\title{
RADIATION EFFECT ON MHD FREE- CONVECTION FLOW OF A GAS AT A STRETCHING SURFACE WITH A UNIFORM FREE STREAM
}

\author{
AHMED Y. GHALY AND ELSAYED M. E. ELBARBARY
}

Received 26 October 2000 and in revised form 3 March 2001

We investigate the problem of free convection heat transfer near an isothermal stretching sheet. This has been done under the simultaneous action of buoyancy, radiation, and transverse magnetic field. The governing equations are solved by the shooting method. The velocity and temperature functions are represented graphically for various values of the flow parameters: radiation parameter $F$, free convection parameter Gr, magnetic parameter $M$, Prandtl number Pr, and the parameter of relative difference between the temperature of the sheet, and the temperature far away from the sheet $r$. The effects of the radiation and magnetic field parameters on the shear stress and heat flux are discussed.

\section{Introduction}

The study of the boundary layer behaviour on continuous surfaces is important because the analysis of such flows finds applications in different areas such as the aerodynamic extrusion of a plastic sheet, the cooling of a metallic plate in a cooling bath, the boundary layer along material handling conveyers, and the boundary layer along a liquid film in condensation processes. As examples on stretched sheets, many metallurgical processes involve the cooling of continuous strips or filaments by drawing them through a quiescent fluid and that in the process of drawing, when these strips are stretched.

Sakiadis [7], first presented boundary layer flow over a continuous solid surface moving with constant speed. Erickson et al. [4] extended Sakiadis' problem to include blowing or suction at the moving surface 
and investigated its effects on the heat and mass transfer in the boundary layer. Danberg and Fansber [2] investigated the nonsimilar solution for the flow in the boundary layer past a wall that is stretched with a velocity proportional to the distance along the wall. P. S. Gupta and A. S. Gupta [5] studied the heat and mass transfer corresponding to the similarity solution for the boundary layer over an isothermal stretching sheet subject to blowing or suction. Chen and Char [1] investigated the effects of variable surface temperature and variable surface heat flux on the heat transfer characteristics of a linearly stretching sheet subject to blowing or suction. Vajravelu and Hadyinicolaou [9] studied the convective heat transfer in an electrically conducting fluid near an isothermal stretching sheet and they studied the effect of internal heat generation or absorption. Recently, Elbashbeshy [3] investigated heat transfer over a stretching surface with variable and uniform surface heat flux subject to injection and suction.

All the above investigations are restricted to MHD flow and heat transfer problems. However, of late, the radiation effect on MHD flow and heat transfer problems have become more important industrially. At high operating temperature, radiation effect can be quite significant. Many processes in engineering areas occur at high temperatures and a knowledge of radiation heat transfer becomes very important for the design of the pertinent equipment. Nuclear power plants, gas turbines and the various propulsion devices for aircraft, missiles, satellites, and space vehicles are examples of such engineering areas. Takhar et al. [8] studied the radiation effects on MHD free convection flow for a non gray-gas past a semi-infinite vertical plate.

In this paper, we propose investigating the radiation effect on steady free convection flow near isothermal stretching sheet in the presence of a magnetic field. The resulting coupled nonlinear ordinary differential equations are solved by shooting methods. A solution for the velocity and temperature functions are obtained. The shear stress and heat flux have been computed.

\section{Analysis}

Here we consider the flow of an electrically conducting fluid, adjacent to a vertical sheet coinciding with the plane $y=0$, where the flow is confined to $y>0$. Two equal and opposite forces are introduced along the $x$-axis (see Figure 2.1), so that the sheet is stretched keeping the origin fixed. A uniform magnetic field of strength $B_{0}$ is imposed along the $y$ axis. The fluid is considered to be a gray, absorbing emitting radiation but non-scattering medium and the Rosseland approximation [6] is used to describe the radiative heat flux in the energy equation. The radiative 


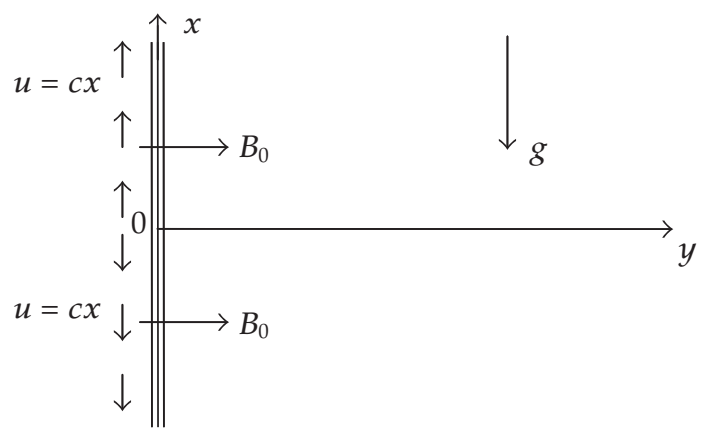

FIGURE 2.1. Sketch of the physical model.

heat flux in the $x$-direction is considered negligible in comparison to the $y$-direction.

Under the usual boundary layer approximation, the flow and heat transfer in the presence of radiation are governed by the following equations:

$$
\begin{aligned}
\frac{\partial u}{\partial x}+\frac{\partial v}{\partial y} & =0 \\
u \frac{\partial u}{\partial x}+v \frac{\partial u}{\partial y} & =v \frac{\partial^{2} u}{\partial y^{2}}+g \beta\left(T-T_{\infty}\right)-\frac{\sigma B_{0}^{2}}{\rho} u \\
u \frac{\partial T}{\partial x}+v \frac{\partial T}{\partial y} & =\frac{k}{\rho c_{p}} \frac{\partial^{2} T}{\partial y^{2}}-\frac{1}{\rho c_{p}} \frac{\partial q_{r}}{\partial y},
\end{aligned}
$$

where $u$ and $v$ are the velocity components in the $x$ and $y$-directions, respectively, $T$ is the temperature, $g$ is the acceleration due to gravity, $v$ is the fluid kinematics viscosity, $\rho$ is the density, $\sigma$ is the electric conductivity, $\beta$ is the coefficient of thermal expansion, $k$ is the thermal conductivity, $c_{p}$ is the specific heat at constant pressure, and $q_{r}$ is the radiative heat flux. The boundary conditions of the problem are

$$
\begin{array}{ll}
u=c x, \quad v=0, \quad T=T_{w} & \text { at } y=0, \\
u \longrightarrow u_{\infty}, \quad T \longrightarrow T_{\infty} & \text { as } y \longrightarrow \infty,
\end{array}
$$

where $c>0, T_{w}$ is the constant temperature of sheet, $T_{\infty}$ is the temperature far away from the sheet, and $u_{\infty}$ is the free stream velocity. By using the Rosseland approximation [6], we have

$$
q_{r}=-\frac{4 \sigma^{*}}{3 k^{*}} \frac{\partial T^{4}}{\partial y},
$$


where $\sigma^{*}$ is the Stefan-Boltzmann constant and $k^{*}$ is the mean absorption coefficient. By using (2.5), the energy equation (2.3) becomes

$$
u \frac{\partial T}{\partial x}+v \frac{\partial T}{\partial y}=\frac{k}{\rho c_{p}} \frac{\partial^{2} T}{\partial y^{2}}+\frac{4 \sigma^{*}}{3 k^{*} \rho c_{p}} \frac{\partial^{2} T^{4}}{\partial y^{2}}
$$

Introducing the following nondimensional parameters:

$$
\begin{gathered}
\bar{x}=\frac{c x}{u_{\infty}}, \quad \bar{y}=\frac{c y R}{u_{\infty}}, \quad \bar{u}=\frac{u}{u_{\infty}}, \\
\bar{v}=\frac{v R}{u_{\infty}}, \quad \theta=\frac{T-T_{\infty}}{T_{w}-T_{\infty}},
\end{gathered}
$$

we can obtain the governing equation in dimensionless form as (with dropping the bars)

$$
\begin{aligned}
& \frac{\partial u}{\partial x}+\frac{\partial v}{\partial y}=0 \\
& u \frac{\partial u}{\partial x}+v \frac{\partial u}{\partial y}=\frac{\partial^{2} u}{\partial y^{2}}+\operatorname{Gr} \theta-M u \\
& u \frac{\partial \theta}{\partial x}+v \frac{\partial \theta}{\partial y}=\frac{1}{\operatorname{Pr}} \frac{\partial^{2} \theta}{\partial y^{2}}+\frac{4}{3 F \operatorname{Pr}}\left((1+r \theta)^{3} \frac{\partial^{2} \theta}{\partial y^{2}}+3 r(1+r \theta)^{2}\left(\frac{\partial \theta}{\partial y}\right)^{2}\right)
\end{aligned}
$$

with the boundary conditions

$$
\begin{array}{ll}
u=x, \quad v=0, \quad \theta=1 & \text { at } y=0, \\
u=1, \quad \theta=0 & \text { as } y \longrightarrow \infty,
\end{array}
$$

where $M=\sigma B_{0}^{2} / \rho c$ is the magnetic parameter, $R=u_{\infty} / \sqrt{c \mathcal{V}}$ is the Reynolds number, $\mathrm{Gr}=g \beta\left(T_{w}-T_{\infty}\right) / c u_{\infty}$ is the free convection parameter, $\mathrm{Pr}=$ $\mu c_{p} / k$ is the Prandtl number, $F=k k^{*} / 4 \sigma^{*} T_{\infty}^{3}$ is the radiation parameter, $\mu=\rho v$ is the viscosity of the fluid, and $r=\left(T_{w}-T_{\infty}\right) / T_{\infty}$ is the relative difference between the temperature of the sheet and the temperature far away from the sheet.

Introducing the stream function $\Psi$ defined in the usual way

$$
u=\frac{\partial \Psi}{\partial y}, \quad v=-\frac{\partial \Psi}{\partial x}
$$


equation (2.9) can then be written as

$$
\begin{aligned}
\frac{\partial \Psi}{\partial y} \frac{\partial^{2} \Psi}{\partial x \partial y}-\frac{\partial \Psi}{\partial x} \frac{\partial^{2} \Psi}{\partial y^{2}}= & \frac{\partial^{3} \Psi}{\partial y^{3}}-M \frac{\partial \Psi}{\partial y}+\mathrm{Gr} \theta \\
\frac{\partial \Psi}{\partial y} \frac{\partial \theta}{\partial x}-\frac{\partial \Psi}{\partial x} \frac{\partial \theta}{\partial y}= & \frac{1}{\operatorname{Pr} \frac{\partial^{2} \theta}{\partial y^{2}}} \\
& +\frac{4}{3 F \operatorname{Pr}}\left((1+r \theta)^{3} \frac{\partial^{2} \theta}{\partial y^{2}}+3 r(1+r \theta)^{2}\left(\frac{\partial \theta}{\partial y}\right)^{2}\right)
\end{aligned}
$$

and the boundary conditions (2.10) become

$$
\begin{array}{ll}
\frac{\partial \Psi}{\partial y}=x, \quad \frac{\partial \Psi}{\partial x}=0, \quad \theta=1 & \text { at } y=0, \\
\frac{\partial \Psi}{\partial y}=1, \quad \theta=0 & \text { as } y \longrightarrow \infty .
\end{array}
$$

Introducing,

$$
\Psi(x, y)=f(y)+x g(y),
$$

in (2.12) and equating coefficients of $x^{0}$ and $x^{1}$, we obtain the coupled nonlinear ordinary differential equations

$$
\begin{gathered}
f^{\prime \prime \prime}=f^{\prime} g^{\prime}-g f^{\prime \prime}+M f^{\prime}-\mathrm{Gr} \theta, \\
g^{\prime \prime \prime}=g^{\prime 2}-g g^{\prime \prime}+M g^{\prime}, \\
\left(3 F+4(1+r \theta)^{3}\right) \theta^{\prime \prime}+3 \operatorname{Pr} F g \theta^{\prime}+12 r(1+r \theta)^{2} \theta^{\prime 2}=0 .
\end{gathered}
$$

The primes above indicate differentiation with respect to $y$ only. The boundary conditions (2.13) in view of (2.14) is reduced to

$$
f(0)=f^{\prime}(0)=g(0)=g^{\prime}(\infty)=\theta(\infty)=0, \quad g^{\prime}(0)=\theta(0)=f^{\prime}(\infty)=1 .
$$

The physical quantities interested in this problem are the skin friction coefficient and the Nusselt number which are defined by

$$
\tau_{w}=\left.\mu\left(\frac{\partial u}{\partial y}\right)\right|_{y=0}, \quad N_{u}=\frac{q_{w}}{k\left(T_{w}-T_{\infty}\right)}
$$

where

$$
q_{w}=-\left.k \frac{\partial T}{\partial y}\right|_{y=0}
$$


98 Radiation effect on MHD free-convection flow

TABLE 2.1. Variation of $f^{\prime \prime}, g^{\prime \prime}, \theta^{\prime}$ at the plate surface with $F, \mathrm{Gr}, M$, and Pr parameters.

\begin{tabular}{llllccl}
\hline$F$ & Gr & $M$ & $\operatorname{Pr}$ & $g^{\prime \prime}(0)$ & $\theta^{\prime}(0)$ & $f^{\prime \prime}(0)$ \\
\hline 1 & 0.5 & 0.1 & 0.73 & $-1.04771(-1.04881)$ & -0.224411 & 0.820805 \\
2 & 0.5 & 0.1 & 0.73 & $-1.04771(-1.04881)$ & -0.297402 & 0.703769 \\
3 & 0.5 & 0.1 & 0.73 & $-1.04771(-1.04881)$ & -0.335702 & 0.656791 \\
\hline 1 & 0 & 0.1 & 0.73 & $-1.04771(-1.04881)$ & -0.224411 & 0.110292 \\
1 & 0.5 & 0.1 & 0.73 & $-1.04771(-1.04881)$ & -0.224411 & 0.820805 \\
1 & 1 & 0.1 & 0.73 & $-1.04771(-1.04881)$ & -0.224411 & 1.53188 \\
\hline 1 & 0.5 & 0.01 & 0.73 & $-1.00398(-1.00499)$ & -0.230155 & 1.12575 \\
1 & 0.5 & 0.1 & 0.73 & $-1.04771(-1.04881)$ & -0.224411 & 0.820805 \\
1 & 0.5 & 0.5 & 0.73 & $-1.22325(-1.22474)$ & -0.204004 & 0.513629 \\
\hline 1 & 0.5 & 0.1 & 0.73 & $-1.04771(-1.04881)$ & -0.224411 & 0.820805 \\
1 & 0.5 & 0.1 & 2 & $-1.04771(-1.04881)$ & -0.480357 & 0.523724 \\
1 & 0.5 & 0.1 & 5 & $-1.04771(-1.04881)$ & -0.882528 & 0.36651 \\
\hline
\end{tabular}

Using (2.14), the quantities in (2.19) can be expressed as

$$
\tau_{w}=\mu c R\left(f^{\prime \prime}(0)+x g^{\prime \prime}(0)\right), \quad N_{u}=\frac{c R}{u_{\infty}} \theta^{\prime}(0) .
$$

The effect of the parameters $F, \mathrm{Gr}, M$, and $\operatorname{Pr}$, on the functions $f^{\prime \prime}, g^{\prime \prime}$, and $\theta^{\prime}$ at the plate surface is tabulated in Table 2.1 for $r=0.05$.

\section{Numerical procedure}

The shooting method for linear equations is based on replacing the boundary value problem by two initial value problems, and the solution of the boundary value problem is a linear combination between the solutions of the two initial value problems. The shooting method for the nonlinear boundary value problem is similar to the linear case, except that the solution of the nonlinear problem cannot be simply expressed as a linear combination between the solutions of the two initial value problems. Instead, we need to use a sequence of suitable initial values for the derivatives such that the tolerance at the end point of the range is very small. These sequences of initial values are given by the secant method. The numerical computations have been done by the 
symbolic computation software Mathematica. The fourth-order RungeKutta method is used to solve the initial value problems. The number of grid points is 1000 and a value of $y_{\max }$, the edge of the boundary layer, ranging from 10 to 15 .

The numerical approach is carried out in two stages. Equation (2.16) has to be solved by the nonlinear shooting method to obtain $g$. Hence, equations (2.15) and (2.17) reduce to a system of linear equations with variable coefficients which could be solved by the linear shooting method to obtain $f$ and $\theta$. The functions $f^{\prime}, g^{\prime}$, and $\theta$ are shown in Figures 3.1, 3.2, and 3.3.

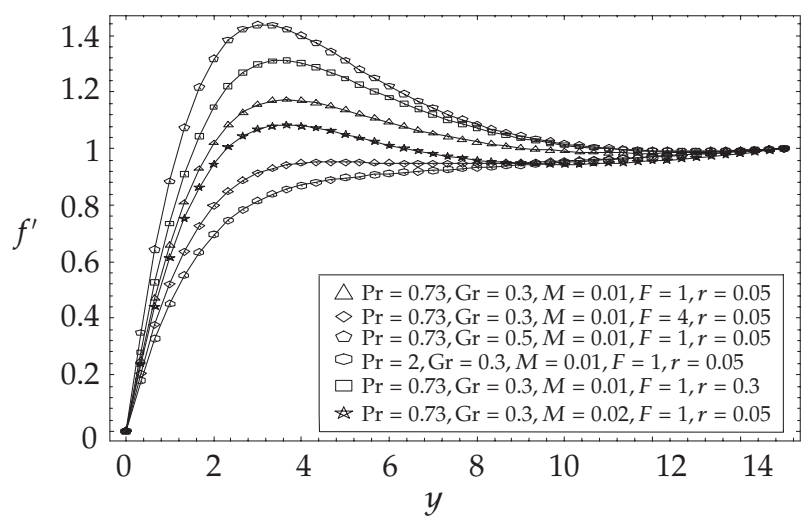

FIGURE 3.1. Variation of the dimensionless velocity component $f^{\prime}$ with $\mathrm{Pr}, \mathrm{Gr}, M, r$, and $F$ parameters.

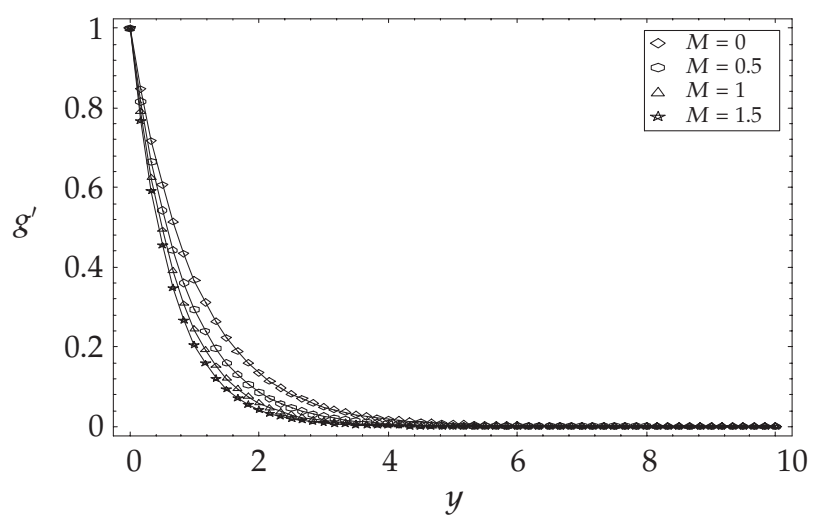

FIGURE 3.2. Variation of the dimensionless velocity component $g^{\prime}$ with $M$ parameter. 


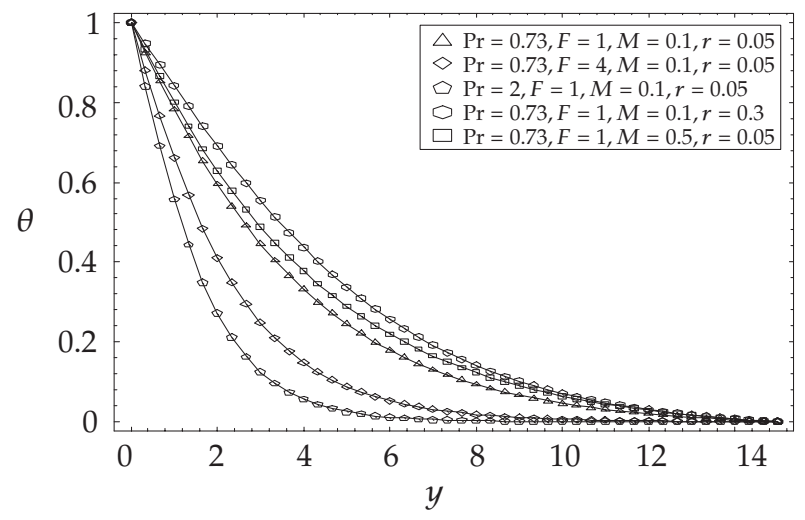

FIGURE 3.3. Variation of the dimensionless temperature $\theta$ with $\operatorname{Pr}, M, r$, and $F$ parameters.

\section{Results and discussion}

The system of differential equations (2.15), (2.16), and (2.17) governed by the boundary condition (2.18) was solved numerically by using the shooting method. It is observed here that radiation does affect the velocity and temperature field of free convection flow of an electrically conducting fluid near isothermal stretching sheets in the presence of a transverse magnetic field. Velocity components $f^{\prime}$ and $g^{\prime}$ as well as the temperature $\theta$ distribution are presented in Figures 3.1, 3.2, and 3.3 for various values of radiation parameter, magnetic field parameter, Prandtl number, and Grashof number. Figure 3.1 shows the variation of $f^{\prime}$ for several sets of values of the dimensionless parameters $F, \mathrm{Pr}, \mathrm{Gr}, r$, and $M$. Moreover, Figure 3.1 shows that $f^{\prime}$ decreases with increasing the radiation parameter $F$ and Prandtl number Pr. It is seen, as expected, that $f^{\prime}$ decreases with increasing the magnetic field parameter $M$. As $M$ increases, the Lorentz force, which opposes the flow, also increases and leads to enchanted deceleration of the flow. This result qualitatively agrees with the expectations, since the magnetic field exerts a retarding force on the free convection flow. However, $f^{\prime}$ increases with an increase in Grashof number $\mathrm{Gr}$ and the parameter of relative difference between the temperature of the sheet and the temperature far away from the sheet $r$. Figure 3.2 describes the behavior of $g^{\prime}$ with changes in the values of the magnetic field parameter $M$. It is seen, as expected, that $g^{\prime}$ decreases with increasing the magnetic field parameter $M$. The effects of the parameters $\operatorname{Pr}, M, F$, and $r$ on the heat transfer are shown in Figure 3.3. It is observed that the temperature increases with an increase in $r$ and $M$ parameters. It is seen that the temperature $\theta$ decreases as the 
radiation parameter $F$ increases. This result qualitatively agrees with expectations, since the effect of radiation is to decrease the rate of energy transport to the fluid, thereby decreasing the temperature of the fluid. It is also observed that the temperature decreases with an increase in the Prandtl number Pr. This is in agreement with the physical fact that the thermal boundary layer thickness decreases with increasing Pr.

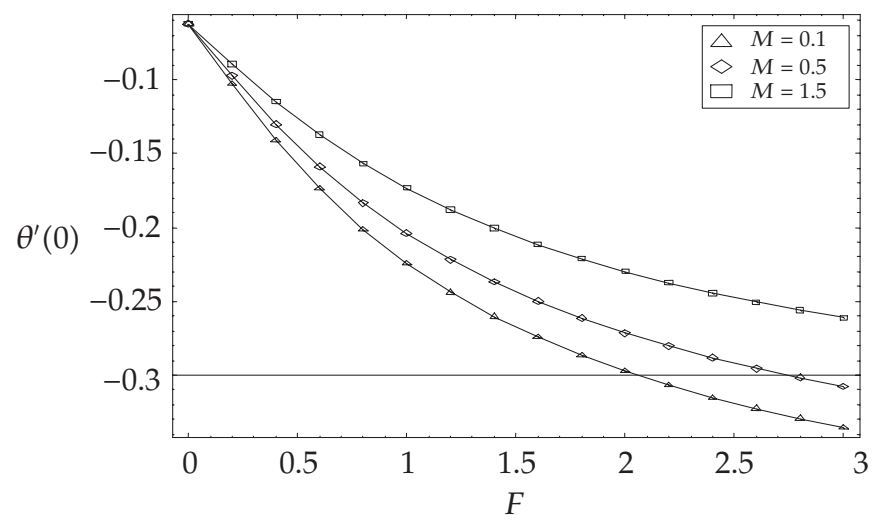

FIGURE 4.1. Variation of the heat flux $\theta^{\prime}(0)$ with $F$ and $M$ parameters.

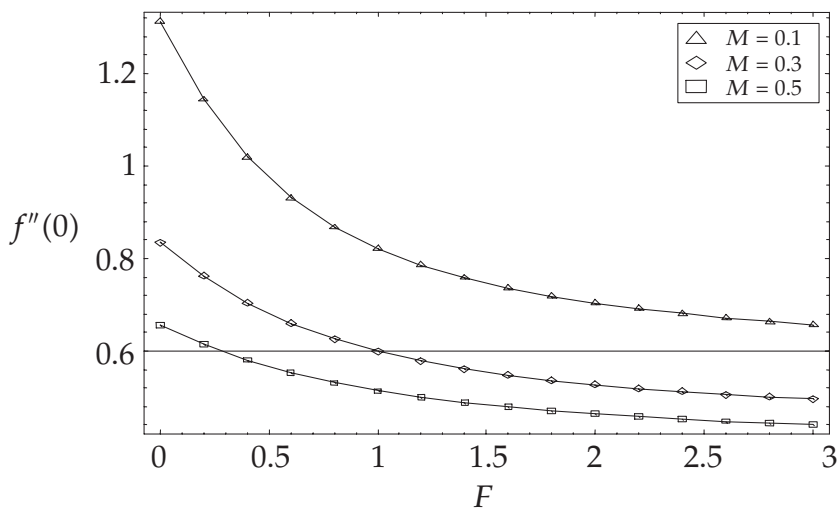

FIGURE 4.2. Variation of $f^{\prime \prime}(0)$ with $F$ and $M$ parameters.

Figures 4.1 and 4.2 describe the behavior of $f^{\prime \prime}(0)$ and the heat flux $\theta^{\prime}(0)$ with changes in the values of the flow parameters $F$ and $M$. We observe that the effect of increasing $M$ is the decrease in the wall 
temperature gradient $\theta^{\prime}(0)$ and $f^{\prime \prime}(0)$. On the other hand, the magnitude of $\theta^{\prime}(0)$ increases and $f^{\prime \prime}(0)$ decreases as $F$ increases. Furthermore, the negative values of the wall temperature gradient, for all values of the parameters, are indicative of the physical fact that the heat flows from the sheet surface to the ambient fluid.

Finally, in order to verify the proper treatment of the present problem, we will compare the obtained numerical solution with the exact values of $g^{\prime \prime}(0)$. The exact solution of $(2.16)(g(y)=-v)$ is given by

$$
g(y)=\frac{1}{\sqrt{M+1}}\left(1-e^{-\sqrt{M+1} y}\right) .
$$

In Table 2.1, the given numbers between brackets refer to the exact values and the given numbers without brackets refer to the approximated values. Vajravelu and Hadyinicolaou [9] have obtained for $g^{\prime \prime}(0)(M=0.01)$ the value of -1.0025 , while our result is -1.00398 and the exact value is -1.00499 . Therefore, the present results are in satisfactory agreement with the exact values.

\section{References}

[1] C. K. Chen and M. I. Char, Heat transfer of a continuous, stretching surface with suction or blowing, J. Math. Anal. Appl. 135 (1988), no. 2, 568-580.

[2] J. E. Danberg and K. S. Fansber, Nonsimilar solution for the flow in the boundary layer past a stretched wall, Quart. Appl. Math. 34 (1976), 305-311.

[3] E. M. A. Elbashbeshy, Heat transfer over a stretching surface with variable surface heat flux, J. Phys. D: Appl. Phys. 31 (1998), 1951-1954.

[4] L. E. Erickson, L. T. Fan, and V. G. Fox, Heat and mass transfer on a moving continuous flat plate with suction or injection, Indust. Eng. Chem. 5 (1966), 19-25.

[5] P. S. Gupta and A. S. Gupta, Heat and mass transfer on a stretching sheet with suction or blowing, Canadian J. Chem. Engrg. 55 (1977), 744-746.

[6] W. M. Rohsenow, J. P. Hartnett, and Y. I. Cho (eds.), Handbook of Heat Transfer, 3rd ed., McGraw-Hill, New York, 1998.

[7] B. C. Sakiadis, Boundary layer behaviour on continuous solid surfaces: I. The boundary layer equations for two-dimensional and axisymmetric flow, AIChE J. 7 (1961), 26-28.

[8] H. S. Takhar, R. S. R. Gorla, and V. M. Soundalgekar, Radiation effects on MHD free convection flow of a gas past a semi-infinite vertical plate, Internat. J. Numer. Methods Heat Fluid Flow 6 (1996), no. 2, 77-83.

[9] K. Vajravelu and A. Hadjinicolaou, Convective heat transfer in an electrically conducting fluid at a stretching surface with uniform free stream, Internat. J. Engrg. Sci. 35 (1997), no. 12-13, 1237-1244.

Ahmed Y. Ghaly: Department of Mathematics, Faculty of Education, Ain Shams University, Roxy, Heliopolis, Cairo, Egypt 
A. Y. Ghaly and E. M. E. Elbarbary 103

Elsayed M. E. Elbarbary: Department of Mathematics, Faculty of Education, Ain Shams University, Roxy, Heliopolis, Cairo, Egypt

Current address: Department of Mathematics, Al Jouf Teacher College, Al Jouf, Skaka, P.O. Box 269, Saudi Arabia

URL: http://elbarbary.cjb.net

E-mail address: eelbarbary@hotmail.com 


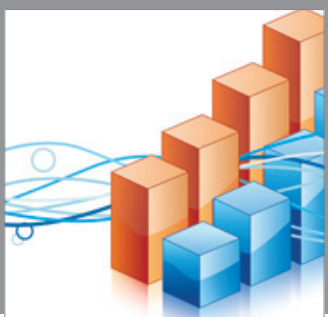

Advances in

Operations Research

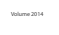

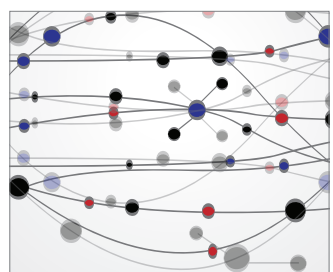

\section{The Scientific} World Journal
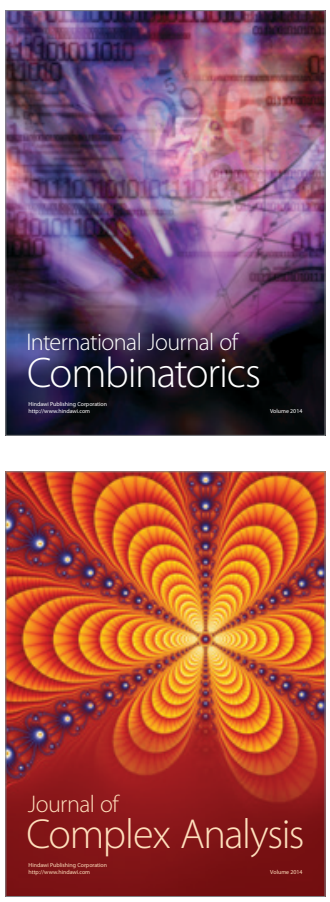

International Journal of

Mathematics and

Mathematical

Sciences
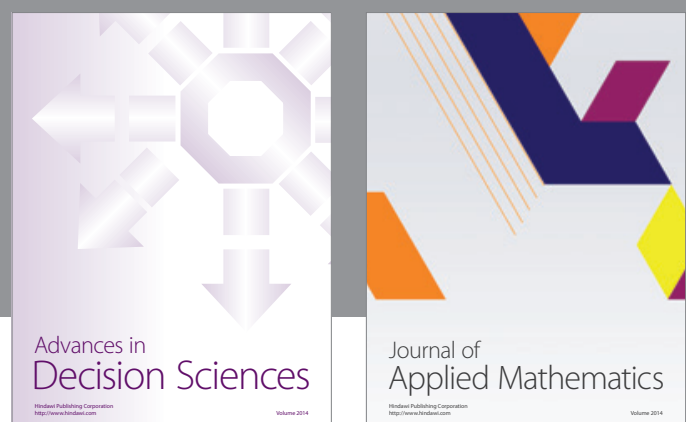

Journal of

Applied Mathematics
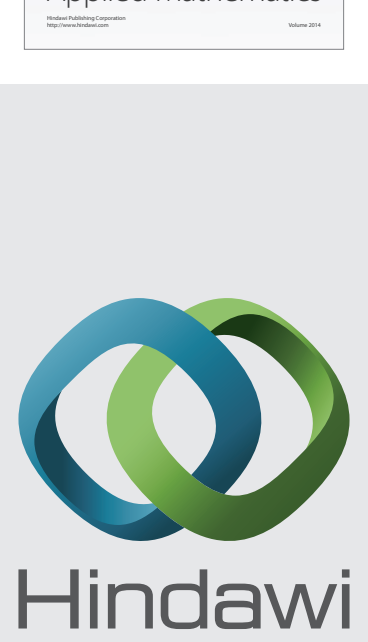

Submit your manuscripts at http://www.hindawi.com
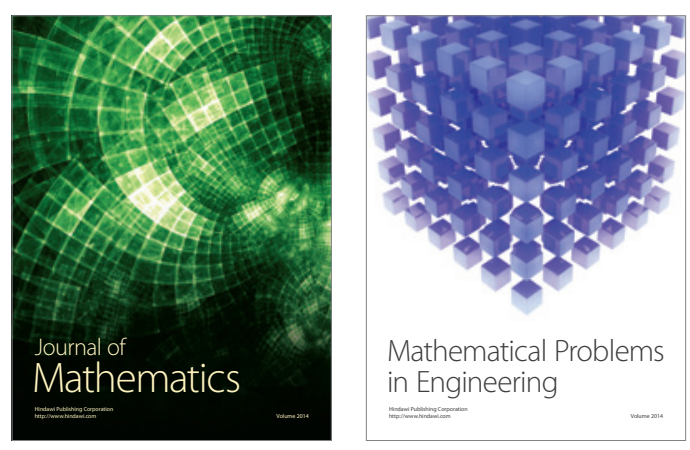

Mathematical Problems in Engineering
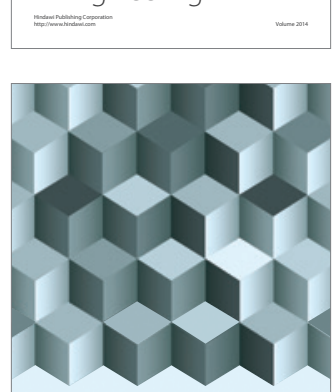

Journal of

Function Spaces
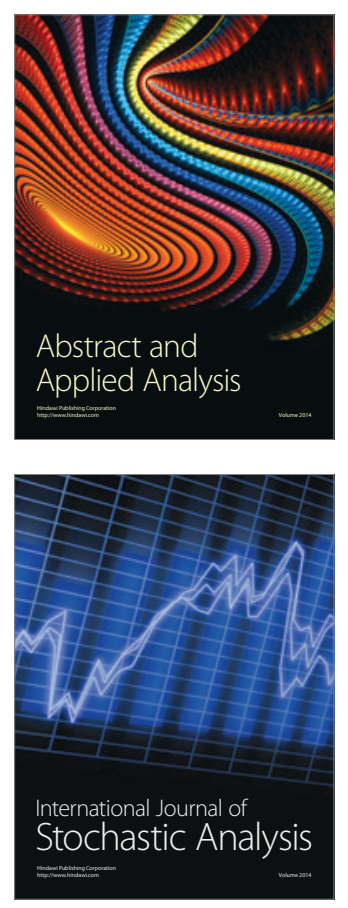

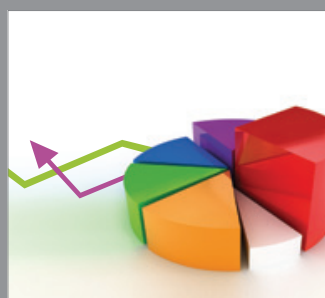

ournal of

Probability and Statistics

Promensencen
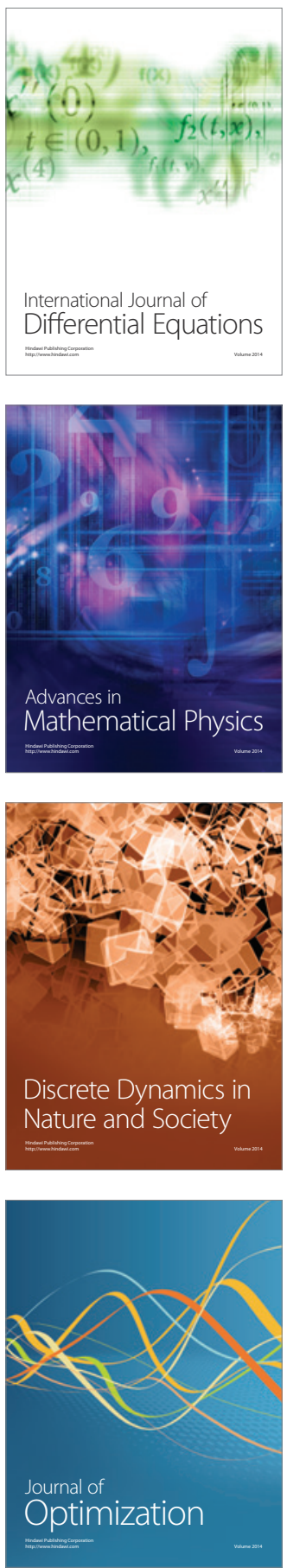